\title{
A Strategy for Atherosclerotic Lesions Segmentation
}

\author{
Roberto Rodríguez and Oriana Pacheco \\ Digital Signal Processing Group, \\ Institute of Cybernetics, Mathematics \& Physics (ICIMAF) \\ rrme.icmf.inf.cu
}

\begin{abstract}
The watersheds method is a powerful segmentation tool developed in mathematical morphology, which has the drawback of producing oversegmentation. In this paper, in order to prevent its over-segmentation, we present a strategy to obtain robust markers for atherosclerotic lesions segmentation of the thoracic aorta. In such sense, we introduced an algorithm, which was very useful in order to obtain the markers of atherosclerotic lesions. The obtained results by using our strategy were validated calculating the false negatives (FN) and false positives (FP) according to criterion of pathologists, where $0 \%$ for $\mathrm{FN}$ and less than $11 \%$ for FP were obtained. Extensive experimentation showed that, using real image data, the proposed strategy was very suitable for our application.
\end{abstract}

\section{Introduction}

Segmentation and contour extraction are important steps towards image analysis. Segmented images are now used routinely in a multitude of different applications, such as, diagnosis, treatment planning, localization of pathology, study of anatomical structure, computer-integrated surgery, among others. However, image segmentation remains a difficult task due to both the variability of object shapes and the variation in image quality. Particularly, medical images are often corrupted by noise and sampling artifacts, which can cause considerable difficulties when applying rigid methods.

The pathological anatomy is a speciality where the use of different techniques of digital image processing (DIP) allows to improve the accuracy of diagnosis of many diseases. One of the most important diseases to study is the atherosclerosis and its organic-consequences, which is one of the principal causes of death in the world [1]. The atherosclerosis produces as final consequence the loss of elasticity and increase of the wall of arteries. For example, heart attack, cerebral attack and ischemia are some of its principal consequences [2].

Many segmentation methods have been proposed for medical-image data [3-6]. Unfortunately, segmentation using traditional low-level image processing techniques, such as thresholding, histogram, and other classical operations, requires a considerable amount of interactive guidance in order to get satisfactory results. Automating these model-free approaches is difficult because of shape complexity, shadows, and variability within and across individual objects. Furthermore, noise and other image artifacts can cause incorrect regions or boundary discontinuities in objects recovered from these methods. 
In mathematical morphology (MM) important methods have been developed for image segmentation [7, 8]. One of the most powerful tools developed in MM is the watersheds transformation, which is classic in the field of topography and it has been used in many problems of image segmentation. However, the watersheds transformation has the disadvantage of producing over-segmentation. For that reason, the correct way to use watersheds for grayscale image segmentation is to mark the regions we want to segment, that is, the objects, but also the background.

The goal of this paper is to present a strategy to obtain robust markers for atherosclerotic lesions segmentation of the thoracic aorta. In such sense, we introduced an algorithm to obtain markers, which identifies correctly the atherosclerotic lesions and eliminates considerably all spurious information. The validity of our strategy was tested by using watersheds segmentation, where the atherosclerotic lesions were correctly delineated according to the criteria of pathologists.

This paper is organized as follows: Section 2 outlines the theoretical aspects and the method of evaluation. In section 3, we present the features of the studied images. In section 4, we introduce an algorithm to obtain the markers. In section 5, we show the validity of our strategy and we carry out a test of the obtained results. Finally, we describe our conclusions in Section 6.

\section{Theoretical Aspects}

This section presents the most important theoretical aspects.

\subsection{Pre-processing}

With the goal of diminishing the noise in the original images we used the Gauss filter. We carried out several researches with many images, arriving to the final conclusion that the best performance are obtained, according to our application, with $\sigma=3$ and a $3 \times 3$ window size. We verified that with these parameters the noise was considerably smoothed and the edges of the interest objects (lesions) were not affected.

\subsection{Contrast Enhancement}

Contrast enhancement is a very used technique as previous step to segmentation. There are many methods in the literature that can be seen $[9,10]$. In this work, we improve the contrast via histogram modification.

\subsection{Morphological Grayscale Reconstruction}

Let $J$ and $I$ be two grayscale images defined on the same domain $D_{I}$, taking their values in the discrete set $\{0,1, \ldots \ldots, L-1\}$ and such that $J \leq I$ (i.e., for each pixel $p \in$ $\left.D_{I}, J(p) \leq I(p)\right)$. $L$ being an arbitrary positive integer. In this way, it is useful to introduce the geodesic dilations according to the following definition [7]:

Definition 2.3.1 (Geodesic dilation). The elementary geodesic dilation of $\delta_{I}^{(1)}(J)$ of grayscale image $J \leq I$ "under" $I$ ( $J$ is called the marker image and $I$ is the mask) is defined as, 


$$
\delta_{I}^{(1)}(J)=(J \oplus B) \wedge I
$$

where the symbol $\wedge$ stands for the pointwise minimum and $J \oplus B$ is the dilation of $J$ by flat structuring element $B$. The grayscale geodesic dilation of size $n \geq 0$ is obtained by,

$$
\delta_{I}^{(n)}(J)=\delta_{I}^{(1)} \circ \delta_{I}^{(1)} \circ \cdots \cdots \circ \delta_{I}^{(1)}(J), n \text { times }
$$

This leads to the following definition of grayscale reconstruction,

Definition 2.3.2 (Grayscale reconstruction). The grayscale reconstruction $\rho_{I}(J)$ of $I$ from $J$ is obtained by iterating grayscale dilations of $J$ "under" $I$ until stability is reached, that is,

$$
\rho_{I}(J)=\bigcup_{n \geq 1} \delta_{I}^{(n)}(J)
$$

Definition 2.3.3 (Geodesic erosion). Similarly, the elementary geodesic erosion $\varepsilon_{I}^{(1)}(J)$ of grayscale image $J \geq I$ "above" $I$ is given by,

$$
\varepsilon_{I}^{(1)}(J)=\left(\begin{array}{l}
J \theta B \\
\theta
\end{array}\right) \vee I
$$

where $\vee$ stands for the pointwise maximum and $J \theta B$ is the erosion of $J$ by flat structuring element $B$. The grayscale geodesic erosion of size $n \geq 0$ is then given by,

$$
\varepsilon_{I}^{(n)}(J)=\varepsilon_{I}^{(1)} \circ \varepsilon_{I}^{(1)} \circ \cdots \cdots \cdot \varepsilon_{I}^{(1)}(J), n \text { times }
$$

Reconstruction turns out to provide a very efficient method to extract regional maxima and minima from grayscale images. Furthermore, the technique extends to the determination of maximal structures, which will be call $h$-domes and $h$-basins. The only parameter $(h)$ is related to the height of these structures. The mathematical background and other definitions can be found in [7].

\subsection{Watersheds Segmentation}

In what follows, we consider grayscale images as numerical functions or as topographic relief.

Definition 2.4.1 (Catchment Basin). The catchment basin $C(M)$ associated with a minimum $M$ is the set of pixels $p$ of $D_{I}$ such that a water drop falling at $p$ flows down along the relief, following a certain descending path called the downstream of $p$, and eventually reaches $M$.

Using the former definitions, it is possible to present the watershed definition. The notion of watershed will now serve as a guideline for the segmentation of grayscale images.

Definition 2.4.2 (Watersheds by Immersion). Suppose that we have pierced holes in each regional minimum of $I$, this picture being regarded as a topographic surface. We then slowly immerse this surface into a lake. Starting from the minimum of lowest altitude, the water will progressively fill up the different catchment basins of $I$. Now, at each pixel where the water coming from two different minima would merge, we build a dam (see Fig. 1). At the end of this immersion procedure, each minimum is completely surrounded by dams, which delimit its associated catchment basin. The whole set of dams which has been built thus provides a tessellation of I in its different catchment basins. These dams correspond to the watershed of I, that is, these represent the edges of objects. 


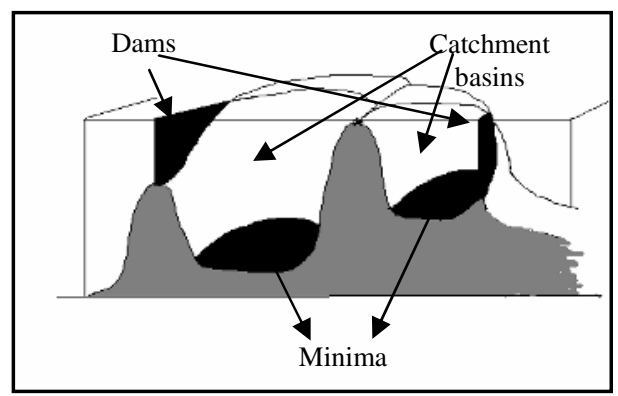

Fig. 1. Building dams at the places where the water coming from two different minima would merge

In many practical cases, one of the principal problems is the obtaining the regional minimum, due to the fact that, in general, images are corrupted by noise. Therefore, the correct way to use watersheds for grayscale image segmentation consists in first detecting markers of the objects to be extracted. When one works in the other way, then the watersheds transformation produces over-segmentation. The oversegmentation mainly comes from the fact that the markers are not perfectly appropriate to the objects to be contoured. In short, the quality of the segmentation is directly linked to the marking function. In this work, the proposed strategy permits to obtain good markers, which were useful for the segmentation process.

\subsection{The Method of Evaluation}

In order to evaluate the performance of the proposed strategy, we calculate the percent of false negatives ( $F N$, atherosclerotic lesions, which are not classified by the strategy) and the false positives ( $F P$, noise, which is classified as atherosclerotic lesion). These are defined according to the following expressions,

$$
\begin{aligned}
& F P=\frac{f_{p}}{V_{p}+f_{p}} * 100 \\
& F N=\frac{f_{n}}{V_{p}+f_{n}} * 100
\end{aligned}
$$

where $V_{p}$ is the real quantity of atherosclerotic lesions identified by the physician, $f_{n}$ is the quantity of atherosclerotic lesions, which were not marked by the strategy and $f_{p}$ is the number of spurious regions, which were marked as atherosclerotic lesions.

\section{Features of the Studied Images}

Studied images are of arteries, which have atherosclerotic lesions and these were obtained from different parts of the human body from more of 80 autopsies. These arteries were contrasted with a special tint in order to accentuate the different lesions in arteries. Later, the lesions are manually classified by the pathologists according to World Health Organization. They classified the lesions in type I, II, III and IV. For 
example, the lesions I and II, these are the fatty streaks and fibrous plaques respectively, while the lesions III and IV are respectively the complicated and calcified plaques. The arteries were digitalized directly from the working desk. It is possible to observe from the images that the different arterial structures are well defined. Other works have used the photograph of the arteries to digitalize the image $[11,12]$. This constitutes an additional step, increases the cost of the research, and leads to a loss of information in the original image. The segmentation process is then more difficult too. These images were captured via the MADIP system with a resolution of 512x512x8 bit/pixels [13].

There are several remarkable characteristics of these images, which are common to typical images that we encounter in the atherosclerotic lesions:

1. High local variation of intensity is observed both, within the atherosclerotic lesions and the background. However, the local variation of intensities is higher within the lesions than in background regions.

2. The histograms showed that there is a low contrast in the images.

3. The lesions III and IV have better contrast than the lesions I and II

4. It is common of these images the diversity in shape and size of the atherosclerotic lesions.

\section{Experimental Results. Discussion}

It is very important to point out that the proposed strategy was obtained according to experimentation, that is, we carried out firstly a morphological reconstruction by erosion for each of the lesions, and secondly, we carried out a morphological reconstruction by dilation for each of the lesions. We verified that in all cases the best results for the lesions I and II using a reconstruction by dilation were obtained, while for the lesions III and IV the obtained results were much better for a reconstruction by erosion.

With the goal of extracting the approximate regions of interest, after the histogram modification, we carried out a morphological reconstruction. We verified that the reconstruction by erosion (for the lesions III and IV) led to an image where the dark zones correspond to these lesions. For example, in Fig. 2 is shown the obtained result for a lesions IV.

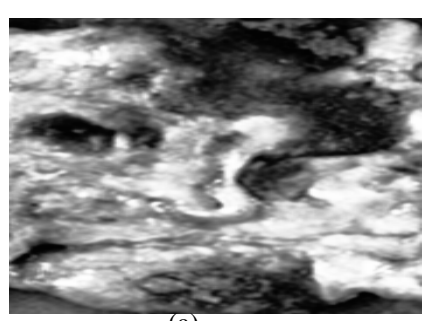

(a)

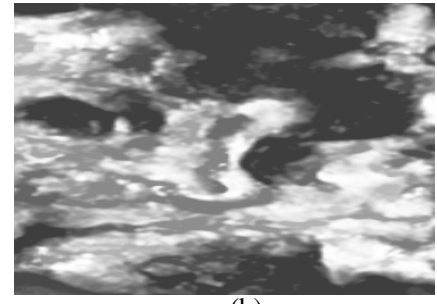

(b)

Fig. 2. (a) Resulting image of the histogram modification. (b) Image obtained by a reconstruction by erosion. The dark parts correspond to the lesion IV. 
The result in Fig. 2(b) was obtained by using a rhomb as structuring element of 5x5 pixels and a height equal to 60 . The selection of this structuring element and its size was obtained via experimentation. These values were used for the lesion III too.

We carried out several experiments with different structuring element and with different size, which we did not put here for problem space. With respect to the height, we verified that for each of our images the optimal value was in the range from 40 to 60 .

After obtaining both, the size of structuring element and the optimal height, the next stage of our strategy was to segment the approximate region of interest, that is, a region that contains the atherosclerotic lesions and its neighbouring background. This step was carried out by applying a simple threshold through Otsu method. The thresholding value does not have much influence on the performance, because the exact shape and size of this region are not important, and hence the region is referred to as an approximate region. In Fig. 3(b) one can see the region of interest.

After this result, we introduce the following algorithm to obtain markers for the atherosclerotic lesions.

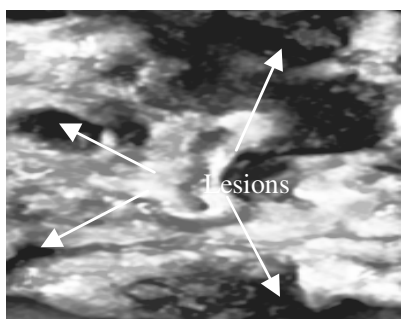

(a)

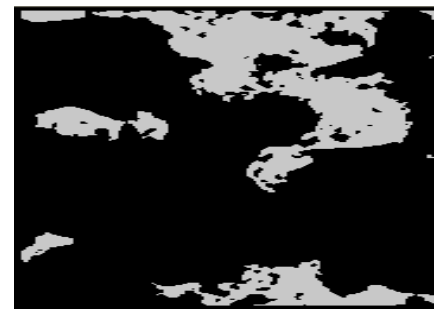

(b)

Fig. 3. (a) Image of the reconstruction, where the arrows indicate the lesions. (b) Regions of interest.

\subsection{Algorithm to Obtain Markers}

The steps of the algorithm are described below:

1. Obtain the regions of interest. Let IREZI be the resulting image.

2. Label the resulting image of the step 1 . Create an auxiliary image; let IA1 be this image. All pixels of this image are put in zero. Scan IREZI at iterative way and all the background in IA1 is labeled with a value equal to 1 .

3. Scan IREZI again from the top to the bottom and from the left to the right. If there is a pixel, which belongs to a connected component and in the image IAl this pixel has zero value, then other iterative method begins to work. This new iterative method marks with a determined value within the image IA1 all pixels belonging to a connected component. In addition, pixels within the image IREZI are also marked with a value, which identifies the connected component to which they belong. As this step is finished, in the image IREZI all the connected components were filled and in the image IA1 all the connected components were labeled.

4. Create other auxiliary image (let IA2 be this image) with the same values of the image IA1. Create also an array, which controls if a connected component was reduced. In the image IA2 is where in each step the reduction of the connected components are obtained, the final result is represented in the image IA1. 
5. Scan the labeled image (IA1). When in this image a pixel is found, which belongs to a connected component, through other iterative method, this component is reduced and in the image IA2 all the frontiers of the connected component are marked. If still there is some pixel within the connected component, which is no frontier, in the images IA 2 and IA1, the mentioned pixel is eliminated and this function begins again until that all points are frontiers. In this case, the obtained result (reduction) is taken as the mark.

6. Finish when the image IREZI is completely scanned. When this step is concluded, in the image IA1 all marks of BV are. These marks are collocated in the image IREZI. Here, after the step two, the connected components (in IREZI) were filled. The image IREZI is the resulting image.

The result of applying this algorithm to the image of Fig. 3(b) is shown in Fig. 4. In Fig. 4(b) one can see that the mark is unique for each of the atherosclerotic lesions, which is always within these. This procedure was carried out for the lesions III and IV.

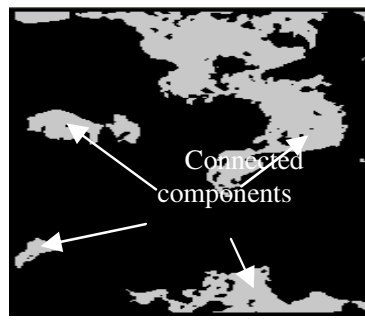

(a)

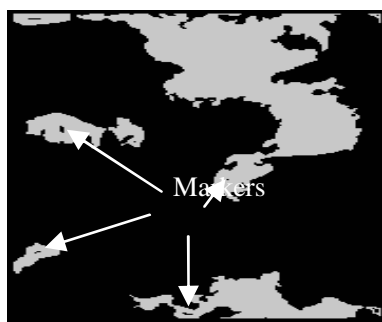

(b)

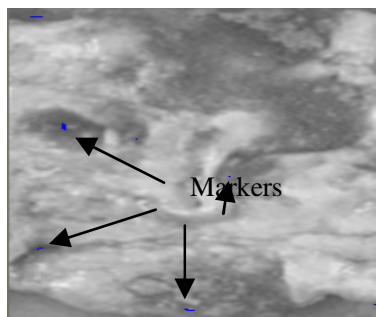

(c)

Fig. 4. (a) Image with regions of interest. (b) Marking image. (c) Marks superimposed on the original image.

Now, we will explain the steps that we carried out to obtain the marks for the lesions I and II. We carried out a reconstruction by dilation. This reconstruction improved more these lesions. Fig.5 shows the obtained result of the reconstruction.

Later, we obtained the approximate region of interest and the markers similarly as in the lesions III and IV. In Fig. 6 is shown the obtained result.

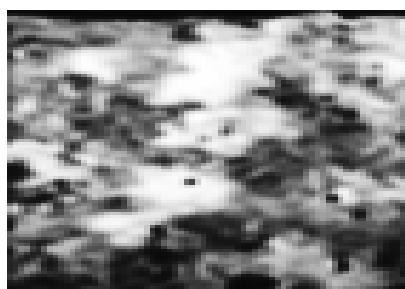

(a)

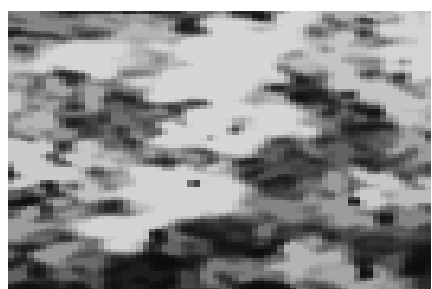

(b)

Fig. 5. (a) Initial image. (b) Reconstruction by dilation (lesion II and II). 


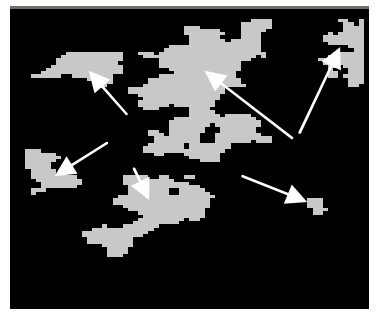

(a)

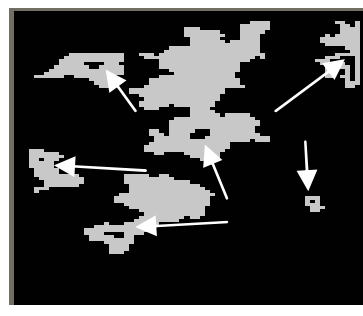

(b)

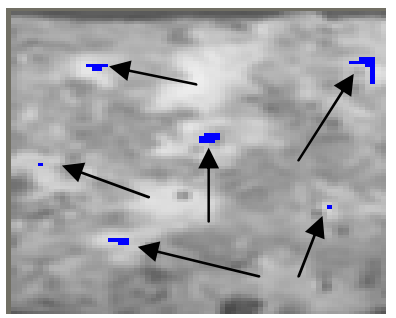

(c)

Fig. 6. (a) Regions of interest. The arrows indicate the connected components. (b) Image with marks. (c) The marks superimposed on the original image.

\section{Application of the Proposed Strategy for Atherosclerosis Image Segmentation by Using the Watershed Method}

As we have pointed out the watershed transformation has the drawback of producing an over-segmentation as it is applied directly to the original image or the gradient image. In fact, Fig. 7(b) shows the obtained result as we applied directly the watershed transformation to an atherosclerosis image without good markers. However, in Fig. 7(c) is shown the excellent result obtained according to our strategy and the introduced algorithm in this work. The contours of the atherosclerotic lesions were well defined.

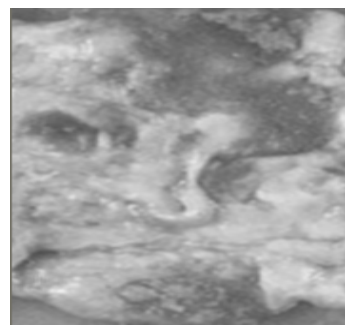

(a)

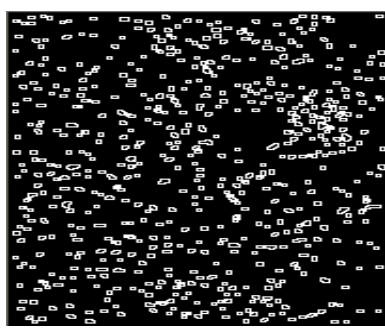

(b)

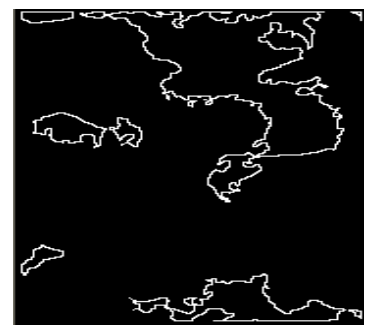

(c)

Fig. 7. (a) Original image. (b) The watershed segmentation without marks in the lesions. (c) The watershed segmentation according to our strategy.

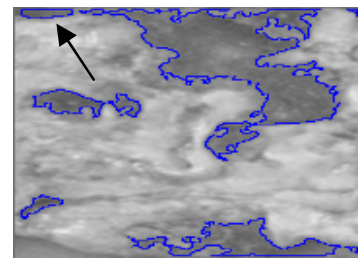

Fig. 8. The contours superimposed on the original image. The arrow indicates an object, which does not correspond to an atherosclerotic lesion. 


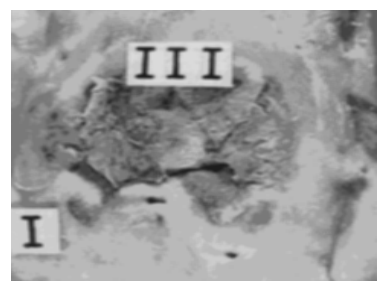

(a)

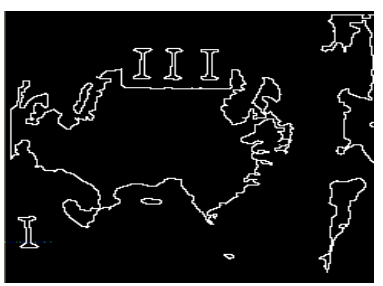

(b)

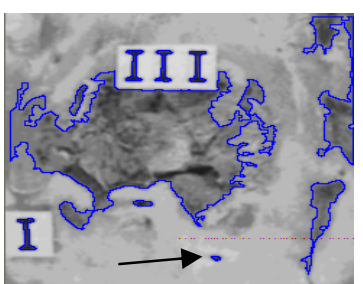

(c)

Fig. 9. (a) Original image. (b) Watershed transformation. (c) Contours superimposed on the original image. The arrow in Figure 9 (c) indicates an object, which does not belong to the lesion.

In Fig. 8, we show the contours superimposed on the original image in order to see the exact coincidence of the obtained contours. This result is evident

In Fig. 9, another example, with real image, of the application of our strategy is shown. The authors have several examples, which we did not present for problem space

\section{Conclusions}

In this work, we proposed a strategy to obtain robust markers for atherosclerotic lesions. In such sense, we introduced an algorithm, which identifies correctly the atherosclerotic lesions and all undesirable information is considerably eliminated. With our strategy the application of the watersheds transformation provided excellent results, and we obtained the exact contours of the atherosclerotic lesions. We showed by extensive experimentation by using real image data, that the proposed strategy was robust for the type of images considered. This strategy was tested, according to the criteria of pathologists, obtaining the false negatives (FN) and false positives (FP), where the percent for $\mathrm{FN}$ was equal to $0 \%$ and for FP minor than $11 \%$. The results were obtained for more of 80 images.

\section{References}

1. Fernández-Britto, J. E., Carlevaro, P. V., Bacallao, J., Koch, A.S., Guski,H., Campos, R.: "Coronary Atherosclerotic lesion: Its study applying an atherometric system using discriminant analysis". Zentralbl.allg. Pathol. 134: 243-249, 1988.

2. Cotran, Robbins: Patología Estructural y Funcional, Mc. Grow Hill, México, ISBN: 97010-2787-6, 2002.

3. W. Kenong, D. Gauthier and M. D. Levine, "Live Cell Image Segmentation", IEEE Transactions on Biomedical Engineering, vol.42, no. 1, enero 1995.

4. J. Sijbers, P. Scheunders, M. Verhoye, A. Van der Linden, D. Van Dyck, E. Raman, "Watershed-based segmentation of $3 D$ MR data for volume quatization", Magnetic Resonance Imaging, vol. 15, no. 6, pp 679-688, 1997.

5. C. Chin-Hsing, J. Lee, J. Wang and C. W. Mao, "Color image segmentation for bladder cancer diagnosis", Mathl. Comput. Modeling, vol. 27, no. 2, pp. 103-120, 1998. 
6. P. Schmid, "Segmentation of digitized dermatoscopic images by two-dimensional color clustering”, IEEE Trans. Med. Imag., vol. 18, no. 2, Feb, 1999.

7. Vincent, L.: "Morphological grayscale reconstruction in Image Analysis: Applications and Efficient Algorithms". IEEE Transactions on Image Processing, vol.2, pp. 176-201, April, 1993.

8. Vicent, L and Soille, P.: "Watersheds in digital spaces: An efficient algorithm based on immersion simulations”, IEEE Transact. Pattern Anal. Machine Intell., 13:583-593; 1991.

9. Fuh C-S, Maragos P., Vincent L.: Region based approaches to visual motion correspondence. Technical Report HRL, Harvard University, Cambridge, MA, 1991.

10. Roberto R. M.: “ The interaction and heuristic knowledge in digital image restoration and enhancement. An intelligent system (SIPDI)”, Ph.D Thesis, Institute of Technology, Havana, 1995.

11. Svindland, A. and Walloe, L.: "Distribution pattern of sudanophilic plaques in the descending thoracic and proximal abdominal human aorta". Atherosclerosis, 57: 219-224, 1985.

12. Cornill, J. F., Barrett, W. A., Herderick, E. E., Mahley, R. W. and Fry, D. L.: "Topographic study of sudanophilic lesions in cholesterol-fed minipigs by image analysis". Arteriosclerosis, 5: 415-426, 1985.

13. Rodríguez, R., Alarcón, T. and Sánchez, L.: "MADIP: Morphometrical Analysis by Digital Image Processing", Proceedings of the IX Spanish Symposium on Pattern Recognition and Image Analysis, Vol. I, pp. 291-298, ISBN 84-8021-349-3, 2001, Spain. 